Iustitia Socialis. Revista Arbitrada de Ciencias Jurídicas.

Año IV. Vol. IV. Nº 1. Edición Especial 2019

Hecho el depósito de Ley: FA2016000064 ISSN: 2542-3371

FUNDACIÓN KOINONIA (F.K). Santa Ana de Coro, Venezuela

Juan Ramón Pérez Carrillo

http://dx.doi.org/10.35381/racji.v4i1.544

\title{
La teoría de las fuentes del Derecho. Dimensiones conceptuales y terminológicas
}

The theory of the sources of law. Conceptual and terminological dimensions

\author{
Juan Ramón Pérez Carrillo \\ iramoncuba@gmail.com \\ Universidad San Gregorio de Portoviejo, Manabí \\ Ecuador \\ https://orcid.org/0000-0002-2145-9799
}

Recibido: 30 de octubre de 2019

Aprobado: 03 de diciembre de 2019

\section{RESUMEN}

El presente estudio tiene como cometido realizar un examen y una aproximación al tema de los fundamentos metodológicos de las fuentes del derecho, en particular, se hace referencia a algunas delimitaciones conceptuales, también sobre las diferentes clasificaciones metodológicas. Cierto es que, para la comprensión de las fuentes del derecho, existen elementos claves sin los cuales es imposible realizar un análisis certero sobre la ciencia jurídica.

Descriptores: Fuentes del derecho; fuentes formales; ordenamiento jurídico; análisis jurídico.

\section{ABSTRACT}

The purpose of this study is to carry out an examination and an approach to the subject of the methodological foundations of the sources of law, in particular reference is made to some conceptual delimitations, also about the different methodological classifications. It is true that for the understanding of the sources of law, there are key elements without which it is impossible to make an accurate analysis of legal science.

Descriptors: Sources of law; formal sources; legal system; legal analysis. 


\section{PLANTEAMIENTO INICIAL DEL TEMA}

La cuestión de las fuentes del derecho, aún hoy día continúa sosteniendo dimensiones especiales, pues la citada institución jurídica se ubica dentro de las construcciones dogmáticas y teóricas con trascendencia en los procesos creación y aplicación del derecho, en cualquiera de los contextos normativos que existen en la actualidad. En consecuencia, el presente estudio tiene como propósito principal sistematizar los fundamentos metodológicos sobre las fuentes del derecho, en lo esencial, a un abordaje en el plano argumentativo general y su expresión contemporánea.

\section{DELIMITACIONES CONCEPTUALES}

De inicio podemos plantear que la expresión fuentes del Derecho es ubicada en una larga y sistemática tradición doctrinal que la avala, en un sentido, de acuerdo con Bobbio (1999) una concepción tradicional o clásica sobre las fuentes del derecho, y en otro sentido una concepción racional y justificativa de las mismas, las que fueron desarrolladas con posterioridad por Aguiló Regla (2000), llegando a plantearse que dicha expresión ha sido utilizada con diversos significados, que corre el peligro de convertirse en una institución del derecho de escasa operatividad (Cueto de la Rúa, 1982), llegado el punto que algunos autores como Santamaría Pastor (1998): "el concepto de fuente del Derecho constituye uno de los elementos más confusos y perturbadores de cuantos manejan las ciencias del Derecho positivo (...)".

Sin embargo, no pocos críticos, entre ellos Pérez Luño (2011) centran sus criterios en la utilidad de esta institución a partir de la carencia de una efectividad normativa particular derivada de su inclusión o no resultante del proceso normativo en la categoría de las fuentes del derecho, en ese sentido las preocupaciones fundamentales surgen a partir del concepto de derecho que encierra la expresión.

Siendo así, el abordar dichas fuentes, parece aludir a que ellas implican algo diferente al derecho mismo y que este es únicamente lo que emana de las mismas. La perspectiva dogmático-jurídica sobre las fuentes del Derecho puede interesarse expresamente por ellas una vez formalizadas y, por lo tanto, puede desprenderse de toda preocupación sobre las causas y fundamentos sociales y políticos de los que la 
fuente en sentido formal no es más que su resultado último (Vallet de Goytisolo, 1982).

Las dimensiones aquí esbozadas no persiguen dilucidar la incógnita sobre la naturaleza del derecho o de su contenido, problemáticas que pese a la creencia generalizada de que no tienen que ver con las fuentes del derecho, están íntimamente relacionadas con dicha teoría, por cuanto la definición de las fuentes del derecho es, en últimas, la lucha por decir el derecho, el cual redunda en la lucha por la supremacía del campo jurídico, que a su vez está relacionado con la superioridad de los campos sociales que están regidos por normas jurídicas (Diez Picazo, 1975: 136).

Este proceso involucra una realidad que normalmente percibimos en forma inconsciente, pero que por ello no deja de ser cierta: la lucha que opera en el campo jurídico no es por la eficacia o por la justicia, sino por el monopolio por decir lo que es el derecho. Tampoco se debe perder de vista que las fuentes del derecho revisten una gran trascendencia ideológica y jurídica pues en cualquier sistema jurídico es este un problema eminentemente político y no simplemente conceptual o teórico. En el fondo se esconde una teoría orientada a privilegiar determinadas fuentes como parte de una lucha de poder para decidir sobre el derecho.

De esta forma, la configuración de dichas fuentes repercute entonces en la distribución del poder público dentro del Estado y además guarda relación con los deberes y derechos constitucionales de los ciudadanos, ya que ellas son la expresión jurídico-formal de la distribución del poder político entre los órganos encargados de la creación normativa (Balaguer Callejón, 1992). Conforme a la organización de cada sociedad se determinará el sistema de derecho y la relevancia que se otorga a una fuente (y no a otra), o el proceder los operadores jurídicos en el momento de su aplicación einterpretación.

De forma general, el análisis que se le realiza al sistema de las fuentes del Derecho constituye una de las más importantes instituciones jurídicas y que es estudiada por juristas desde diversas áreas del conocimiento, por ejemplo:

1) Dentro de la Teoría del Derecho, propuesta por, Peces-Barba en el año 2000 quien establece que es una ciencia que dedica su estudio a la disposición interna y 
externa de los ámbitos jurídicos y su relación con la formación de los sistemas jurídicos;

2) El campo del Derecho Constitucional, confirmado por gran parte de la doctrina, tiene a Enrique Álvarez Conde (2008) como figura principal. Actualmente parece común que la regulación de las fuentes del derecho se encuentre en el entorno de la teoría de la Constitución, debido a la naturaleza de fuente del derecho que ha adquirido la norma fundamental;

3) El ámbito del Derecho Civil en los casos que opera dentro del Derecho común supletorio a diferencia del resto del sistema jurídico.

Abordar las fuentes del derecho desde las anteriores tres dimensiones no es exclusivo de estos sectores jurídicos, el ámbito que abarca la teoría de las fuentes del derecho los trasciende a todos ellos, sin que lo anterior sea un impedimento para examinar del mismo modo a las fuentes del derecho en relación con la casuística particular de cada rama del ordenamiento jurídico.

De manera racional, al enfrentar la institución de las fuentes desde un plano teórico amplio, se encuentran cuestiones que guardan relación con presupuestos de lo jurídico, tal como la esencia del derecho o el argumento del mismo, tópicos que resultan polémicos. Aun así, no quiere decir que la esencia y el argumento del derecho sean diferentes al ámbito reflexivo y aclaratorio de las fuentes del derecho, aun cuando pudiera existir dicha creencia, -a veces generalizada- sobre la cuestión. Al contrario, las cuestiones iusfilosóficas están presentes y por una razón elemental, no es posible desvincularlos teóricamente de las fuentes del derecho: porque como último recurso se dirige a la institución de las fuentes del derecho, tal como lo menciona, R.v. Ihering, es la lucha por el derecho, (Ihering, 1957), es decir, la superioridad de lo jurídico frente a cualquier otro tipo de circunstancias.

De acuerdo a diferentes autores, entre ellos, Pérez Royo (2007), Balaguer Callejón (1992), Guastini (1999), la expresión fuentes del derecho ha sido abordada históricamente, desde una diversidad de usos, sobre lo cual también han escrito autores como Diez Picazzo (1973), De Otto (1993), sobre lo cual, ante la imposibilidad de abordar todos los significados, a los efectos de esta investigación y 
desde una visión generalista, indicamos algunos de los más utilizados, a los efectos que nos permitan definirlas e identificarlas en la praxis jurídica.

Adoptando como postulado la doctrina comúnmente aceptada, nosotros hemos sostenido con anterioridad, que se consideran como fuentes del derecho en sentido formal los actos y hechos jurídicos de un sistema de normas e instituciones desde donde se condicionará la producción de normas, o la producción del derecho objetivo, al respecto se puede consultar Pérez Carrillo (2009a), (2009b), (2010).

\section{CLASIFICACIONES METODOLÓGICAS}

Sobre la cuestión que implica a las clasificaciones de las fuentes del derecho, podemos indicar, entre algunas de ellas, a las que de una $u$ otra forma han tenido una repercusión en el orden metodológico, a saber:

- La que establece su distinción entre fuentes del conocimiento, de producción o autoridad creadora, materiales y fuentes formales.

- En otro orden, las signadas por su escritura: fuentes escritas y no escritas, en las primeras tendrían cabida fuentes como la ley; en tanto, entre las no escritas, se incluyen otras como la costumbre, y cuando no están formuladas en la propia norma escrita, los principios generales del derecho. En todo caso, esta clasificación no tiene más interés que por el hecho de la manifestación de las fuentes en relación con su carácter codificado o escrito o su carácter no codificado o no escrito.

- Las clasificadas de acuerdo a su eficacia jurídica: fuentes directas o indirectas, de las primeras se puede decir, que encierran en sí la norma jurídica, las que determinan la existencia en sí misma de las normas de derecho y se suelen reducir a dos: la costumbre y la ley. Mientras que las indirectas son las que ayudan a la producción y a la comprensión de la norma jurídica, pero sin darle existencia por sí mismas.

Una acotación importante, en este aspecto, es que a los efectos doctrinales las fuentes directas, son consideradas las fuentes del derecho en sentido estricto, pues las indirectas o mediatas, son más bien apreciadas con cierta frecuencia como generadoras del conocimiento de lo jurídico. Así Clemente de Diego (1922) 
denominó a las primeras fuentes reales, en tanto que a las segundas las califica como fuentes del conocimiento del derecho.

- Por su procedencia o destinatario: fuentes específicas y eventuales. Las primeras cuando las normas jurídicas que están destinadas a regular las relaciones jurídicas sobre objetos puntuales. Las segundas pueden contener diversas materias, pues su objeto de regulación puede abarcar materias objeto de cualquier rama del derecho; en consecuencia, el destinatario puede ser diverso.

- Fuentes estatales y fuentes extraestatales. Las primeras suponen la creación directa del derecho por parte del Estado a través de sus propios órganos a los que se les asigna esta función. Las segundas son las fuentes que crean el derecho o lo reconocen a través de la conciencia jurídica general, de las fuerzas sociales o de los ambientes jurídicos.

- La que articula el sistema de fuentes del derecho en diferentes grados: fuentes constitucionales, fuentes primarias o legislativas, fuentes secundarias o reglamentarias y las fuentes terciarias como manifestación de la autonomía de los particulares que establecen reglas válidas erga omnes en ámbitos admitidos por el ordenamiento jurídico. (Pegoraro, 2003: 42).

En torno a las clasificaciones es necesario precisar que de acuerdo al criterio de Norberto Bobbio (1999) la distinción entre fuentes del conocimiento jurídico, fuentes de producción, fuentes materiales y fuentes formales, partiendo de la premisa que, por fuentes del derecho son:

Entendidos aquellos actos y hechos en los cuales se apoya el ordenamiento jurídico para crear las normas jurídicas, que no es más que el propio derecho, sin perder de vista su relación con las leyes sobre la producción jurídica de cada ordenamiento.

Por fuentes del conocimiento se señalan aquellos medios, lugares donde se busca el conocimiento sobre determinado orden jurídico, tales como documentos, instrumentos y otros en los que el derecho se encuentra consignado; resumen en sí la cultura jurídica de un sistema de derecho y el material necesario para la reconstrucción del pasado histórico-jurídico. En la actualidad, además de los libros, documentos, revistas jurídicas, etc., se utilizan medios más modernos como la informática para almacenar información relativa al derecho. 
Con la expresión fuentes de producción, se hace referencia a quiénes pueden o tienen la fuerza para crear normas jurídicas, entendidas como todos los actos y hechos que producen o que son idóneos para producir derecho. Estos serían los sujetos que tienen poder o capacidad de originar normas jurídicas, siendo el principal productor de normas de esta índole el Estado, como único ente que ostenta el poder público político, por vía de los órganos a los que se atribuye esta facultad normativa.

No obstante, Pérez Luño (2011) plantea que en la actualidad se percibe la existencia de nuevas y poderosas fuentes de producción normativa de carácter supraestatal, infraestatal y paraestatal, con la pretensión de desplazar el centro de gravedad de la creación normativa, determinado por la existencia de poderes normativos superiores al Estado como son los organismos internacionales o supranacionales, la ampliación de competencias normativas por parte de los entes sociales intermedios, situados entre el ciudadano y el poder estatal (entes territoriales locales, asociaciones sindicales y deportivas, grupos religiosos, entre otros). Como contraposición a la categoría fuentes formales, suelen identificarse según Fernández Bulté (2001), las denominadas fuentes materiales del Derecho que hacen referencia a: "(...) todos los factores extrajurídicos que inciden sobre la creación de las normas jurídicas".

En este caso, signadas, por circunstancias de carácter material u objetivas, de cuales dimana el derecho, de donde surgen las soluciones jurídicas en cada ordenamiento jurídico, pues en definitiva el derecho emerge de las relaciones sociales, cuestiones que están íntimamente relacionada con la posición iusfilosófica y política que las personas adoptan para la concepción sobre las fuentes del Derecho.

La norma, además de ser un resultado de la actividad del legislador, responde a procedimientos específicos y es una manera de regular la conducta de las personas a través de un imperativo. A tal respecto, las fuentes materiales del Derecho son factores, circunstancias y elementos que condicionan y determinan el contenido de la norma jurídica, también susceptibles de ser identificadas como fuentes metajurídicas o sociológicas. Ejemplo de ellas son: la naturaleza de las cosas, la tradición, el interés social, la opinión popular, etc. 
Como se puede apreciar, el carácter de las fuentes formales del derecho, encierran de una parte la problemática de los procedimientos, las vías y mecanismos, y por otra las instituciones y autoridades que conllevan al nacimiento al derecho.

Por su parte el teórico inglés del derecho, Herbert Hart, advierte la existencia de normas primarias que establecen las conductas que se ordenan, en las que se prohíben o se permiten; a la vez que existen otras normas denominadas normas secundarias, donde se agrupan las normas de reconocimiento, las normas de cambio y las reglas o normas de adjudicación.

Las normas secundarias rigen la producción de las primeras, por ello se estima correcto incluir en el concepto de fuentes formales, tal como plantea Fernández Bulté (2001), no solo los actos, hechos jurídicos y procedimientos, sino que también estos deben ajustarse a las normas de reconocimiento; es decir, que deben ser legítimos en relación a un determinado ordenamiento jurídico, y si son normas que cambian a otras, deben hacerlo según la regla de cambio y por la autoridad correspondiente (según la regla de adjudicación).

Como las fuentes formales del derecho identifican el modo de producción normativa de un país, las mismas se traducen en aquellas normas jurídicas que establecen el órgano competente y el procedimiento a través del cual se crean las normas jurídicas. Por ejemplo, en la doctrina italiana las normas sobre la producción jurídica se suelen denominar como metanormas; se trata de normas de un nivel distinto a las creadas por ellas. Esta definición sirve para distinguir entre fuente acto y fuente norma, donde la primera es la ejecución de la segunda, es decir, no existe acto de producción normativa dentro de un sistema jurídico si tal acto no se encuentra autorizado por una metanorma de competencia del mismo sistema jurídico.

A partir de la conocida clasificación que indica a las fuentes del derecho como los actos o hechos a los que el ordenamiento jurídico le atribuye la idoneidad o la capacidad de producir normas jurídicas, nosotros precisamos a los efectos metodológicos de la investigación, la necesidad de indicar en estos casos, tanto a las fuentes legisladas, como a los no legisladas, en el primer caso, son el producto del derecho legislado, el acto normativo en sí, tanto la Constitución como acto normativo extraordinario, como los actos normativos ordinarios, a saber, leyes, 
decretos-leyes, decretos y resoluciones, en el segundo caso, es decir las no legisladas, se incluyen la costumbre, la jurisprudencia y los principios generales del derecho.

De acuerdo a lo antes esbozado, tienen relevancia singular las siguientes cuestiones que hacen referencia a las dimensiones conceptuales sobre las fuentes del derecho siguientes: 1) La determinación de los contenidos formales y materiales de las fuentes del derecho; 2) La subjetividad que nos brinda la información de un superior que genera la fuente, y la metodología que rige sus funciones; 3) El carácter constitucional que reviste a las fuentes del derecho.

De forma sintética podemos señalar algo que se ha convertido en un elemento metodológico orientador, pues resume que la Constitución se despliega en tres dimensiones en cuanto a las fuentes del derecho: por una parte, la Constitución como norma suprema (un instrumento necesario para la racionalización y control de las funciones del Estado); por otra la Carta Magna como elemento indispensable del derecho; y por la último la Constitución como ente regulador y delimitante de las fuentes del derecho.

\section{CONCLUSIONES}

En correspondencia con lo abordado, el abordaje de las fuentes del derecho nos hace remitirnos de manera inexorable a los debates cardinales del derecho y sobre su concepción teórica, siendo esta temática unos de los elementos más controvertidos, para lo cual primordial la posición teórica y filosófica que sobre el derecho se posea, lo cual deriva a su vez en la posición política frente al concepto del derecho.

En ese orden de ideas, a nuestro de evaluar la cuestión, a estas alturas todavía la Constitución continúa siendo el eje fundamental en la construcción del sistema normativo, el cual determina los modos de producción del derecho, por lo cual, en correspondencia con ello, es la norma que ordena el sistema de fuentes del derecho. 
Iustitia Socialis. Revista Arbitrada de Ciencias Jurídicas.

Año IV. Vol. IV. N 1 . Edición Especial 2019

Hecho el depósito de Ley: FA2016000064 ISSN: 2542-3371

FUNDACIÓN KOINONIA (F.K). Santa Ana de Coro, Venezuela

Juan Ramón Pérez Carrillo

\section{REFERENCIAS CONSULTADAS}

1. Aguiló Regla, J. (2000). Teoría General de las Fuentes del Derecho (y el orden jurídico). España: Ariel.

2. Álvarez Conde, E. (2008). Curso de Derecho Constitucional, Vol. I, El Estado Constitucional, el sistema de fuentes, derechos y libertades. España: Tecnos.

3. Balaguer Callejón, F. (1992). Fuentes del Derecho. Vol. I. Principios del ordenamiento constitucional. España: Tecnos.

4. Bobbio, N. (1999). Teoría General del Derecho, (trad. de Jorge Guerrero). Colombia: Temis.

5. Cueto de la Rúa, J. (1982). Fuentes del Derecho. Argentina: Abeledo-Perrot.

6. Clemente de Diego, F. (1922). Las fuentes del Derecho Civil español. Madrid: [s.e.].

7. De Otto, I. (1993). Derecho Constitucional. Sistema de Fuentes. España: Ariel.

8. Diez-Picazo, L. (1973). Experiencias jurídicas y Teoría del Derecho. España: Ariel.

9. Fernández Bulté, J. (2001). Teoría del Estado y el Derecho. Teoría del Derecho. La Habana: Félix Varela.

10. Guastini, R. (1999). "Concepciones sobre las fuentes del Derecho", (trad. de Miguel Carbonell) en Revista Isonomía № 11, México.

11. Ihering, R. (1957). La lucha por el Derecho, (trad. y prólogo de Diego A. de Santillán). México: Cajica.

12. Legaz Lacambra, L. (1961). Filosofía del Derecho. España: Bosch.

13.Peces-Barba, G. (2000). Curso de Teoría del Derecho. España.

14.Pegoraro, Lucio et al. (2003). Las fuentes en el Derecho Comparado (con especial referencia al ordenamiento constitucional) Perú: Universidad Mayor de San Marcos-Instituto Iberoamericano de Derecho Constitucional.

15.Pérez, J. R. (2009a). "Hacia un reconocimiento jurídico-normativo de las fuentes del Derecho en Cuba". en Revista de Derecho. Iuris Veritatis. (1) 1317. 
16.Pérez, J. R. (2009b). "Sobre el tema de las fuentes del Derecho en Cuba". Revista Maestrado em Direito. Direitos Humanos Fundamentais. 9 (2), 91 102.

17.Pérez, J. R. (2010). "Causas de indeterminación en el sistema de fuentes del derecho". Revista Problema. Anuario de Filosofía y Teoría del Derecho, 4, pp. 303-321.

18.Pérez Luño, A. (2011). El desbordamiento de las fuentes del Derecho. España: La Ley.

19.Pérez Royo, J. (2005). Curso de Derecho Constitucional. España: $10^{\mathrm{ma}}$ ed., Marcial Pons.

20. Pérez Royo, J. (2007). Las Fuentes del Derecho. España: Tecnos.

21.Santamaría Pastor, J. (1998): Fundamentos de Derecho Administrativo. España: Centro de Estudios Ramón Areces.

22. Vallet de Goytisolo, J. (1982). "Las expresiones fuentes del Derecho y ordenamiento jurídico", (pp. 825-921) en Estudios sobre Fuentes del Derecho y Método Jurídico. España: Montecorvo.

\section{REFERENCES CONSULTED}

1. Aguiló Regla, J. (2000). General Theory of the Sources of Law (and the legal order). Spain: Ariel.

2. Álvarez Conde, E. (2008). Constitutional Law Course, Vol. I, The Constitutional State, the system of sources, rights and freedoms. Spain: Tecnos.

3. Balaguer Callejón, F. (1992). Sources of Law Vol. I. Principles of constitutional order. Spain: Tecnos.

4. Bobbio, N. (1999). General Theory of Law, (Trad. By Jorge Guerrero). Colombia: Themis.

5. Cueto de la Rúa, J. (1982). Sources of Law Argentina: Abeledo-Perrot.

6. Clemente de Diego, F. (1922). The sources of Spanish Civil Law. Madrid: [s.e.]. 
Iustitia Socialis. Revista Arbitrada de Ciencias Jurídicas.

Año IV. Vol. IV. N 1 . Edición Especial 2019

Hecho el depósito de Ley: FA2016000064 ISSN: 2542-3371

FUNDACIÓN KOINONIA (F.K). Santa Ana de Coro, Venezuela

Juan Ramón Pérez Carrillo

7. De Otto, I. (1993). Constitutional right. Source System Spain: Ariel.

8. Diez-Picazo, L. (1973). Legal experiences and Theory of Law. Spain: Ariel.

9. Fernández Bulté, J. (2001). Theory of State and Law. Law teory. Havana: Felix Varela.

10. Guastini, R. (1999). "Conceptions about the sources of Law", (Trad. By Miguel Carbonell) in Isonomía Magazine № 11, Mexico.

11. Ihering, R. (1957). The fight for the Law, (trad. And prologue of Diego A. de Santillán). Mexico: Cajica.

12. Legaz Lacambra, L. (1961). Philosophy of Law Spain: Bosch.

13. Peces-Barba, G. (2000). Law Theory Course. Spain.

14. Pegoraro, Lucio et al. (2003). Sources in Comparative Law (with special reference to constitutional order) Peru: Universidad Mayor de San MarcosIbero-American Institute of Constitutional Law.

15. Pérez, J. R. (2009a). "Towards a legal-normative recognition of the sources of Law in Cuba." in Law Magazine. luris Veritatis. (1) 13-17.

16. Pérez, J. R. (2009b). "On the subject of the sources of Law in Cuba." Maestrado em Direito Magazine. Fundamental Human Direitos. 9 (2), 91-102.

17.Pérez, J. R. (2010). "Causes of indeterminacy in the system of sources of law." Problem Magazine. Yearbook of Philosophy and Theory of Law, 4, pp. 303-321.

18. Pérez Luño, A. (2011). The overflow of the sources of law. Spain: The Law.

19. Pérez Royo, J. (2005). Constitutional Law Course. Spain: 10th ed., Marcial Pons.

20. Pérez Royo, J. (2007). The Sources of Law. Spain: Tecnos.

21. Santamaría Pastor, J. (1998): Fundamentals of Administrative Law. Spain: Ramón Areces Studies Center. 
Iustitia Socialis. Revista Arbitrada de Ciencias Jurídicas.

Año IV. Vol. IV. ํㅜ 1. Edición Especial 2019

Hecho el depósito de Ley: FA2016000064 ISSN: $2542-3371$

FUNDACIÓN KOINONIA (F.K). Santa Ana de Coro, Venezuela

Juan Ramón Pérez Carrillo

22. Vallet de Goytisolo, J. (1982). "The expressions sources of Law and legal system", (pp. 825-921) in Studies on Sources of Law and Legal Method. Spain: Montecorvo.

(C2019 por los autores. Este artículo es de acceso abierto y distribuido según los términos y condiciones de la licencia Creative Commons Atribución-NoComercial-Compartirlgual 4.0 Internacional (CC BY-NC-SA 4.0) (https://creativecommons.org/licenses/by-nc-sa/4.0/). 